\title{
Cavity enhanced eigenmode multiplexing for volume holographic data storage
}

\section{Bo E. Miller, Yuzuru Takashima}

Bo E. Miller, Yuzuru Takashima, "Cavity enhanced eigenmode multiplexing for volume holographic data storage," Proc. SPIE 10384, Optical Data Storage 2017: From New Materials to New Systems, 1038406 (23 August 2017); doi: 10.1117/12.2272613

EDent: SPIE Optical Engineering + Applications, 2017, San Diego, California, United States 


\title{
Cavity enhanced eigenmode multiplexing for volume holographic data storage
}

\author{
Bo E. Miller* and Yuzuru Takashima \\ The University of Arizona: College of Optical Sciences, 1630 E. University Blvd., Tucson, AZ USA \\ 85721
}

\begin{abstract}
Previously, we proposed and experimentally demonstrated enhanced recording speeds by using a resonant optical cavity to semi-passively increase the reference beam power while recording image bearing holograms. In addition to enhancing the reference beam power the cavity supports the orthogonal reference beam families of its eigenmodes, which can be used as a degree of freedom to multiplex data pages and increase storage densities for volume Holographic Data Storage Systems (HDSS). While keeping the increased recording speed of a cavity enhanced reference arm, image bearing holograms are multiplexed by orthogonal phase code multiplexing via Hermite-Gaussian eigenmodes in a Fe:LiNbO3 medium with a $532 \mathrm{~nm}$ laser at two Bragg angles for expedited recording of four multiplexed holograms. We experimentally confirmed write rates are enhanced by an average factor of 1.1 , and page crosstalk is about $2.5 \%$. This hybrid multiplexing opens up a pathway to increase storage density while minimizing modifications to current angular multiplexing HDSS.
\end{abstract}

Keywords: Multiplexing Holography, Optical Data Storage, Gaussian Eigenmodes, Optical Resonators, Pseudo-phaseconjugate, $\mathrm{Fe}: \mathrm{LiNbO3}$

\section{INTRODUCTION}

As the demand for internet bandwidth continues to increase, both consumers and corporations are exporting their growing data to remote centers. From personal media libraries to corporate finance data it is all being stored remotely. Ideally, we would store all of this data on the fastest possible medium, and have that medium last as long, cost as little, and be as compact as possible. Unfortunately, no such medium exists. Solid State Drives (SSD) are the fastest but most expensive medium, and do not have a good shelf life. Other contenders are magnetic tapes, magnetic Hard Disk Drives (HDD), bit based optical discs, and Holographic Data Storage Systems (HDSS) under research. With so many options, one of the research focuses of data storage is a matter of matching the kind of data with the right medium; this is an issue of "data temperature". The data on a given storage system which is used most frequently is then the hottest data, and it needs to be stored on the fastest storage medium, such as SSD. The rest of the data then falls somewhere between hot and cold, where the coldest data is rarely used but must be kept in storage. For this cold data, it is possible to use slower media since the data is not in high demand during most of its lifetime.

Among the slower media, magnetic tapes and HDDs are the only easily re-writeable media making them suitable for data that falls between hot and cold. Thus HDSS is best suited to archives where Write Once Read Many (WORM) memory is used to store immutable or stable data such as photos, music, medical histories, old financial records, etc. In the WORM field competitors include the SONY/Panasonic Archival Disc (AD) and possibly 5D eternal storage. AD sacrifices data transfer rates-359.65 Mbps - and storage density $-250 \mathrm{~GB} / \mathrm{in}^{3}$ in a standard 12 disc cartridge - for a $\sim 50$ year archive life ${ }^{1}$. 5D storage promises densities as high as $439 \mathrm{~TB} / \mathrm{inch}^{3}$ with an archive life on the order of $10^{20}$ years, but the data rates of this developing technology are still unknown ${ }^{2}$. HDSS, on the other hand, offers maximum data transfer rates of $2.4 \mathrm{Gbps}$ and a storage density of $720 \mathrm{~GB} / \mathrm{in}^{3}$ while maintaining the same $\sim 50$ year archive life as $\mathrm{AD}$ at $1 / 13$ of the total cost of ownership of an $\mathrm{AD}$ system ${ }^{3}$. With all of these advantages, HDSS is a promising option for WORM memory in cold data storage.

While HDSS is a very attractive WORM system, it is not very energy efficient: the nature of the holographic recording and readout process wastes $80 \%$ to $99.99 \%$ of the light provided by its laser source. Moreover, current HDSS storage densities are still well below the theoretical limit. 3D optical data storage is limited to the storage density of $1 / \lambda^{3}$ bits/inch ${ }^{3}{ }^{4}$ which is equivalent to a density on the order of $10 \mathrm{~TB} / \mathrm{inch}^{3}$. Reaching this goal will require the use of many Degrees Of Freedom (DOFs) to increase number of multiplexing. Currently, the number of angular multiplexed holograms is limited by the maximum available angular space for the reference beam, 180 degrees, which is already 
partially occupied by the object angular extent. To further increase capacity in keeping up with improving recording media ${ }^{5}$, additional DOFs are needed to multiplex even more holograms while minimizing the modification of system optics and signal encoding schemes. As demonstrated previously, resonant optical cavities can be employed in readout ${ }^{6}$ and recording ${ }^{7}$ to recover much of the conventionally wasted light allowing for effective gains in energy efficiency, data transfer rates, and storage densities. Cavities can also sustain orthogonal modes for reference beams according to their eigenmodes. These eigenmodes are an inherently orthogonal set of functions, which means that they can be used as orthogonal phase codes to providing another DOF in HDSS multiplexing ${ }^{8-10}$, bringing it closer to the 3D limit when effectively combined with angular multiplexing.

In this paper we report the experimental demonstration of a cavity enhanced, orthogonal mode-angular hybrid multiplexing, which enables HDSS to accesses more multiplexing DOFs. First, cross-talk of image bearing holograms recorded via a Hermite-Gaussian (HG) 0,0 and a mostly-phase Spatial Light Modulator (SLM) generated HG 1,0 reference beam is evaluated. Second, eigenmode multiplexing of two distinct images at single location is demonstrated. Third, to demonstrate cavity enhanced eigenmode recording, images are recorded using a cavity enhanced HG 1,0 reference beam. Finally, we demonstrate cavity enhanced mode-angular hybrid multiplexing by enhancing the write rate of four distinct images which are successfully recorded and reconstructed by using both angle and mode multiplexing. Sections 2-5 address the experimental demonstration of single page crosstalk, eigenmode multiplexing, eigenmode enhancement of write rate, and cavity enhanced mode-angle multiplexing, respectively. Section 6 discusses crosstalk, the utility of the technology in enhancing data density, and potential limitations of cavity enhanced mode multiplexing when it is applied to HDSS along with angular multiplexing.

\section{CROSS-TALK OF SINGLE HOLOGRAMS READ OUT BY ORTHOGONAL CAVITY EIGENMODES}

The orthogonality of image bearing holograms is tested by using HG, optical cavity, eigenmodes as reference beams. We record a single image bearing hologram at its own location in the crystal by either an HG 0,0 or an HG 1,0 reference beam. The image is reconstructed with one of the two orthogonal beams and the diffraction efficiencies are compared when the hologram is read out with each beam. Cross-talk is evaluated by taking the ratio of the diffraction efficiency of the matched reference beam to that of the orthogonal reconstruction beam.

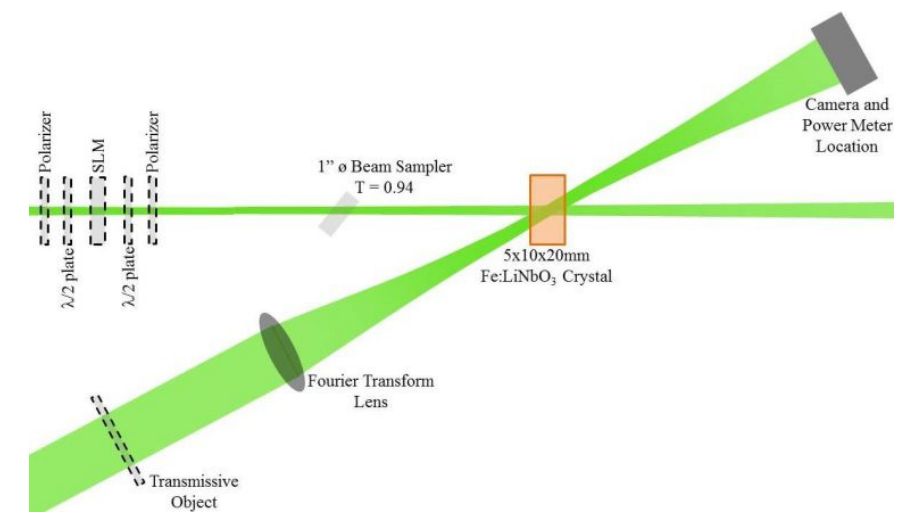

Fig. 1. Experimental setup for evaluating the cross-talk of single holograms.

As seen in Fig. 1 an SLM is setup in the reference arm of a holographic recording geometry and illuminated with a 532 $\mathrm{nm}$ wavelength beam from a frequency doubled Nd:YAG laser. The recording setup is downstream of a lens which Fourier Transforms (FT) the reference beam from the SLM. The linear polarizers and $\lambda / 2$ plates around the SLM are set to operate the SLM in the "mostly phase" modulation mode ${ }^{11}$. The reference beam then passes through the beam sampler, which is used in the trials of Section 4 for enhancement monitoring. The signal arm expands a Gaussian beam to a collimated beam with an approximate diameter of $5 \mathrm{~mm}$. This collimated signal beam passes through the transmissive object to be recorded. The transmissive object is then Fourier Transformed by a microscope objective lens. The two beams intersect inside of a 0.015 mole $\% \mathrm{Fe}: \mathrm{LiNbO}_{3}$ crystal with its c-axis perpendicular to the reference beam 
and parallel to the plane of the recording beams. Upon readout of the hologram a CMOS camera is placed in the readout beam to record the reconstructed image. Diffraction efficiency is also measured at this point with a power meter.

The mode of the reference beam is controlled by setting the SLM to either a vertically split screen or a uniform screen. The split screen creates a 0 to $\pi$ phase step that bisects the incident Gaussian beam converting it to a HG 1,0 beam. The uniform screen uses the SLM to make sure that the reference beam has the same power inside the Fe:LiNbO3 crystal. This is possible because of the "mostly phase" modulation of the SLM which has some amplitude modulation coupled to the phase. The profiles of the reference beams at the location of the Fe:LiNbO3 crystal depicted in Fig. 1 are shown in Fig. 2.

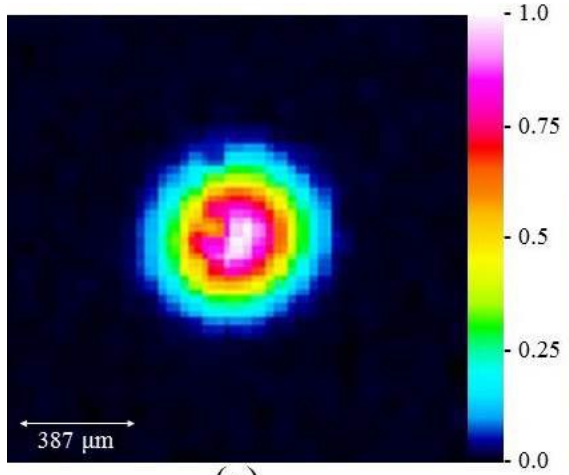

(a)

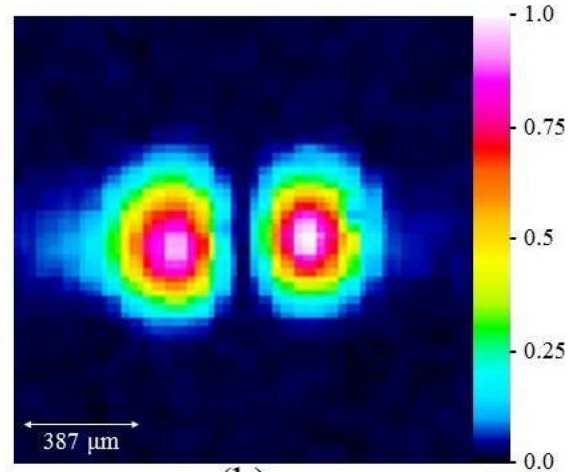

(b)

Fig. 2. Beam profiles for (a) the Gaussian reference beam and (b) the HG 1,0 reference beam at a location of recording material.

The largest number '2' on a resolution bar target (USAF-1951 RES-1, Newport) is used as the object to be recorded. The original image is taken with the CMOS camera and the image is written once with a HG 0,0 reference beam and once at a separate location with a HG 1,0 beam. The holograms are read out via both the HG 0,0 and 0,1 beam and the images are captured. The images can be seen in Fig. 3.

The diffraction efficiency of each hologram is recorded when illuminated with each reference beam. Taking the ratio of those diffraction efficiencies we find that recording with the HG 0,0 beam has a single page cross-talk of $2.5 \%$ and the HG 0,1 recording has a cross-talk of $1.7 \%$. Furthermore, this test was repeated with six other holograms at separate locations in the crystal for a total of eight holograms. Cross-talks were similar for HG 0,0 and 0,1 recording, so we report the mean of all eight cross-talks as $2.48 \%$ with a standard deviation of $1.17 \%$.

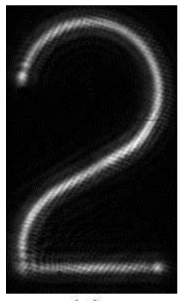

(a)

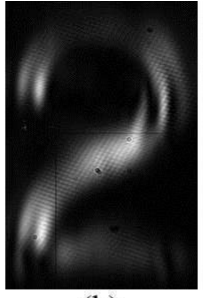

(b)

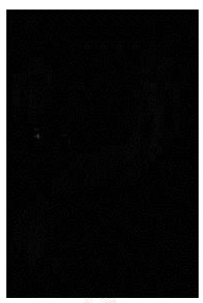

(d)

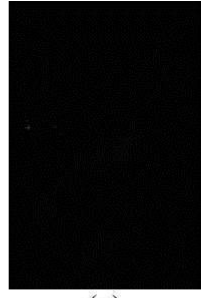

(c)

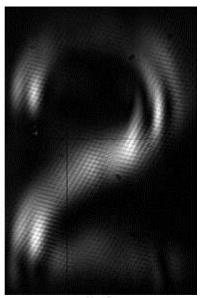

(e)

Fig. 3. Images of the (a) original object recorded; (b) readout by an HG 0,0 beam of a hologram written with a HG 0,0 beam; (c) readout by an HG 1,0 beam of a hologram written with an HG 0,0 beam; (d) readout by an HG 0,0 beam of a hologram written with an HG 1,0 beam; (e) readout by an HG 1,0 beam of a hologram written with an HG 1,0 beam. 


\section{IMAGE MULTIPLEXING WITH HERMITE-GAUSSIAN REFERENCE BEAMS}

Using the experimental setup of Fig. 1, an image of the largest number ' 0 ' on the USAF test chart is recorded using a HG 0,0 reference beam, and an image of the largest number ' 1 ' is recorded at the same location using a HG 1,0 reference beam. The exposure times are adjusted to equalize the diffraction efficiencies of the two holograms. After recording, the two holograms are illuminated by each of the two reference beams, and the resulting images are captured.

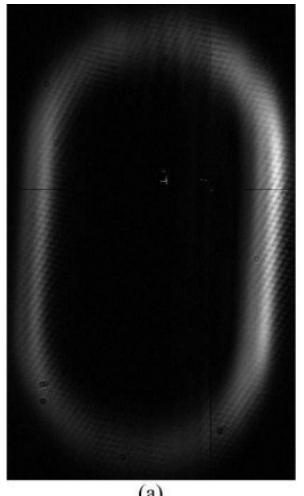

(a)

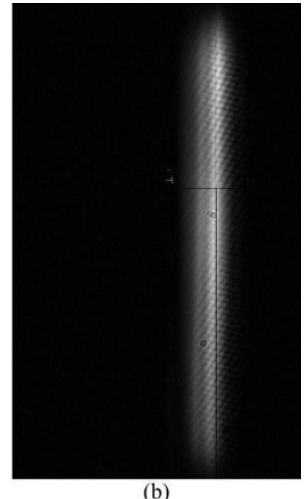

(b)

Fig. 4. Reconstructed images from HG mode multiplexing. (a) Image of the number ' 0 ' reconstructed with the HG 0,0

beam. (b) Image of the number ' 1 ' reconstructed with the HG 1,0 beam.

Fig. 4 shows the captured images from the multiplexing experiment. This clearly demonstrates the low cross-talk of HG mode multiplexing as the ' 1 ' does not show up in the ' 0 ' image and vice versa. In this case we would expect cross-talks similar to those measured in Section 2.

\section{CAVITY ENHANCED WRITING WITH HERMITE-GAUSSIAN EIGENMODES}

A setup similar to that of Fig. 1 in Section 2 is used with the addition of monitoring the diffraction efficiency by a pseudo phase conjugate geometry ${ }^{7}$. In Fig. 5, a resonator cavity is formed around the crystal by inserting a planar entrance coupler, and a spherical mirror. The reference beam is the HG 1,0 generated by the SLM. The cavity is stabilized during the recording process by a proportional gain feedback loop. The reverse propagating reference beam is used to read out the hologram in the pseudo-phase conjugate geometry, and a beam sampler is used to deflect this diffracted signal into the condensing lens. Passing the condensing lens the signal beam is optically chopped, and detected by a lock-in amplified photo diode. The diffraction efficiency is thus measured as a function of time.

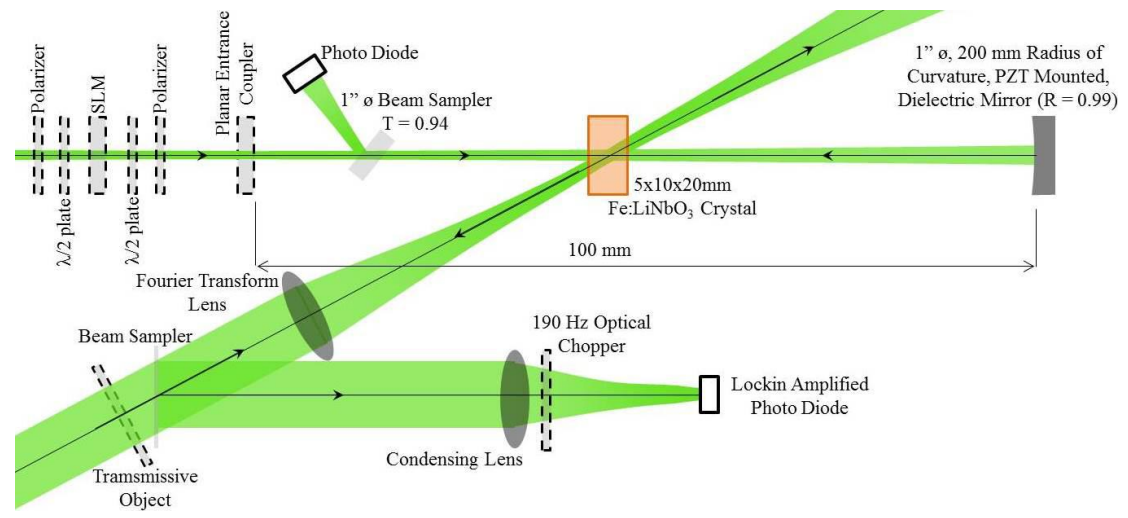

Fig. 5. Experimental setup for cavity enhanced recording with $\mathrm{HG}$ cavity eigenmodes

The time evolutions of the diffraction efficiencies of a cavity and a non-cavity hologram recorded at adjacent locations are then compared by scaling the data to compensate for pseudo-phase conjugate readout and converting the voltage data into actual diffraction efficiency values. This data is fit with Eq. 1 to find the time constant $\tau$ with which the diffraction efficiency grows ${ }^{7}$ : 


$$
\eta_{1}(t)=\sin ^{2}\left(A\left(1-e^{-\frac{t}{\tau}}\right)\right) .
$$

The constant $A$ is a scaling factor related to the available dynamic range, the grating thickness, recording wavelength, and Bragg angle.

Taking the ratio of the non-cavity to cavity time constants we get the cavity enhanced write rate. For five trial pairs we get a Write Rate Enhancement (WRE) of $1.13 \pm 0.03$. The cavity enhancement of irradiance was $\mathrm{G}_{\mathrm{F}}=1.38 \pm 0.07$. With the theoretical WRE being $\sqrt{G_{F}}$, we expect a WRE of $1.18 \pm 0.03$. Thus, we achieved $96 \%$ of the theoretical enhancement with the expected range of theory and result overlapping. This synchronicity proves that HG beams can be used in cavity enhanced recording. The results are summarized in Fig. 6 where the cavity enhanced diffraction efficiency evolves faster than normal writing, and the histogram insert shows that all five trials show enhancement.

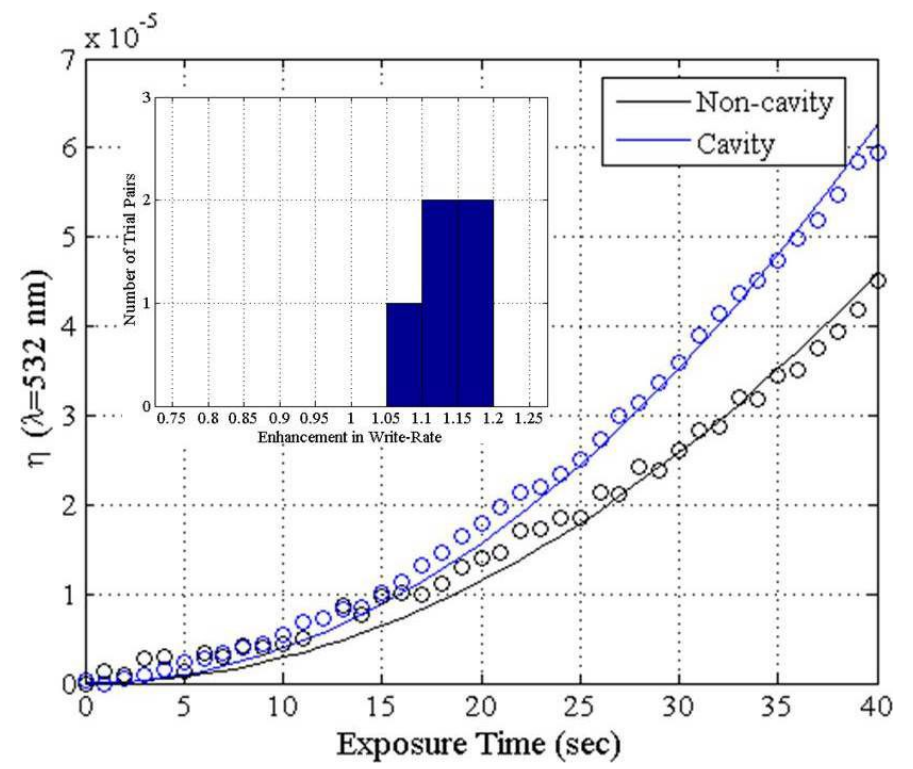

Fig. 6. Data and fitting curves for the best data set including a histogram of the write rate enhancements using a HG 1,0 reference beam. The non-cavity and cavity diffraction efficiency data have a time constants of $1.06 \times 10^{5} \mathrm{sec}$., and $0.909 \times 10^{5}$ sec., which yield a 1.17 enhancement in write data rate. The inset shows a histogram of write rate enhancements for the five trial pairs.

The single page cross-talk was also measured for three of these trials yielding an average cross-talk of $\sim 6.2 \%$. This increase in cross-talk from cavity recording is likely due to poor mode matching to the cavity. The mode matching lens used is sufficient to allow cavity enhancement, but a better choice of mode matching lens would provide better mode purity in the cavity and higher enhancements.

\section{COMBINED ANGULAR AND MODE MULTIPLEXING WITH CAVITY ENHANCED WRITING}

Angular and mode multiplexing are combined while enhancing the write rate with a cavity on the reference arm. The experimental setup is similar to that of Fig. 5, except that the first polarizer, wave plate, and SLM have been replaced with a custom phase plate to convert the beam to a HG 1,0 beam. The Fe:LiNbO3 crystal has also been remounted on a goniometer stage for angular multiplexing. The center of rotation of the goniometer is located at the crossing point of the reference and signal beams. Reconstructions are also observed by placing the CMOS camera where the transmissive object had been for pseudo phase conjugate readout.

Four holograms are multiplexed using two angles, and HG 0,0 and HG 1,0 reference beams. The angular separation was chosen by monitoring the diffraction efficiency of a hologram written with a Gaussian reference beam. While the angle chosen this way is likely still smaller than the first Bragg null, it provides angular cross-talks that are unmeasurable for 
single pages. Each hologram is written with a cavity enhanced reference beam with an average GF $=1.20$. First an image is recorded at $0^{\circ}$ with an $\mathrm{HG} 0,0$ beam, second an image is recorded at $0^{\circ}$ with an $\mathrm{HG} 1,0$ beam, third an image is recorded at $0.6^{\circ}$ with an HG 1,0 beam, and fourth an image is recorded at $0.6^{\circ}$ with an $\mathrm{HG} 0,0$ beam. Each of the four images is reconstructed via pseudo-phase conjugate, cavity enhanced readout, and the results of the reconstruction are shown in Fig. 7.

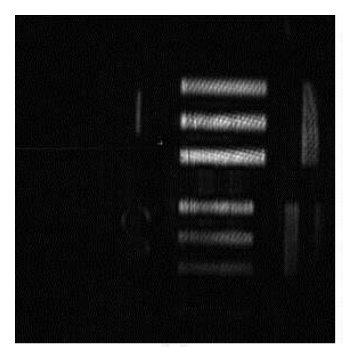

(a)

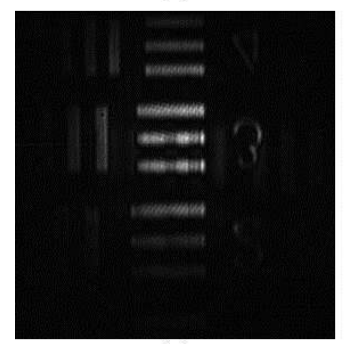

(c)

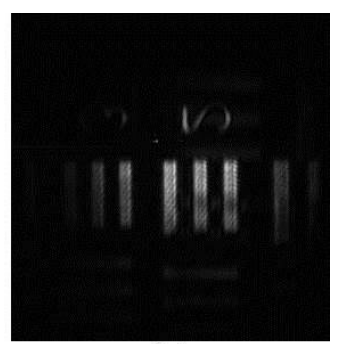

(b)

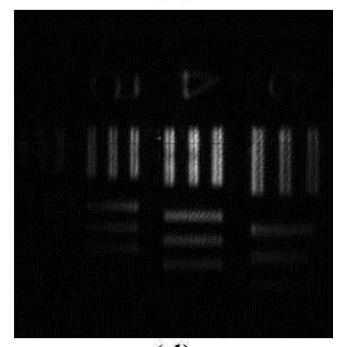

(d)

Fig. 7. Pseudo-phase conjugate reconstruction of image recorded (a) at $0^{\circ}$ with $\mathrm{HG} 0,0$, (b) at $0^{\circ}$ with $\mathrm{HG} 1,0,(\mathrm{c})$ at $0.6^{\circ}$ with $\mathrm{HG} 1,0$, (d) at $0.6^{\circ}$ with $\mathrm{HG} 0,0 . \sim 10 \%$ Cross talk is visible in the reconstructions, and all holograms were written with an average enhancement of $\mathrm{G}_{\mathrm{F}}=1.20$.

In Fig. 7, an average crosstalk of $10 \%$ is observed in the reconstructions, but careful control of cavity mode matching, and mode generation fidelity should remove this effect. This cross-talk is expected to be on the order of $6 \%$ as seen in Section 4, but the change of mode converter is likely responsible for this.

\section{DISCUSSION}

Existing theory predicts zero cross-talk for plane wave signal holograms multiplexed using optical cavity eigenmodes ${ }^{9}$, but we see cross-talks at $\sim 2.5 \%$ in the single page trials of section 2 . This may be due to the cross-talk inherent in page base holography using image bearing object beams ${ }^{8}$, but this cross-talk could also be due to several other factors: SLM phase error, SLM coupled amplitude modulation, Gaussian beam imperfections, and stray light.

The SLM has limited bit levels to choose from for setting the $\pi$ phase shift, so some digitization error may be present in the split screen used to create the HG 1,0 beam. Concurrent to digitization error, the SLM used can only be made to operate in "mostly phase mode", which comes with some amplitude modulation coupled to the phase modulation ${ }^{11,12}$. The combination of these effects are likely responsible for the horizontal spread of the reference beam seen in the HG 1,0 beam profile of Fig. 2, which would decrease the purity of the beam and thus its orthogonality to the Gaussian beam. Similarly, any imperfections in the beam produced by our source would reduce orthogonality.

Cross-talk power readings may also have been influenced by stray light reflecting off of the many surfaces in the system. This is particularly likely as diffracted signals with matched reference and readout beams were on the order of $100 \mathrm{nW}$ while the mismatched signals were on the order of $1 \mathrm{nW}$. The noise of the power meter was around $0.5 \mathrm{nW}$ in the fully dark conditions that data was recorded, so SNR is on the order of 2 for measuring the diffraction efficiency in the mismatched case. This source of error is further supported by the lack of cross-talk seen in the images of Fig. 3.With all of these possible defects it is not surprising that we did not see zero cross-talk, and it is reasonable to expect that careful system design will reduce the cross-talk to more desirable levels.

For the recording of multiplexed images, Bashaw et. all include the effects of dephasing in broadband signal recording and show that orthogonal phase code multiplexing provides overall cross-talk to signal ratios which are a factor of two 
lower than those of angular multiplexing ${ }^{8}$. Such reduced cross-talk is appealing for reducing bit error rates and increasing recording density, but cavity eigenmode multiplexing cannot completely replace angular multiplexing for two reasons.

First, mode dimensions increase as the square root of the mode number, so the cavity diameter puts an upper limit on the number of modes that can be used ${ }^{13}$. Second, when using an FT recording geometry the mode profile of the reference beam affects the modulation transfer function in a manner similar to Gaussian apodization. If the reference mode contains a field null parallel to the signal and reference beam plane of incidence (eg. HG 0,1), the reference beam will act like a high pass filter in recording. This further limits the available choices of cavity modes. Thus, reaching the industry goal of hundreds of multiplexed pages is impossible using only cavity eigenmodes.

Nonetheless, cavity enhanced mode multiplexing combined with angular multiplexing is feasible as seen in Section 6, and is an attractive way to increase data density. Adding mode multiplexing to an angular multiplexing scheme provides an additional DOF for system design increasing data densities toward the theoretical limit ${ }^{4}$. Currently, the number of multiplexed pages reported in literature is about 440 which is primarily limited by the angular extent of the reference beam scanning ${ }^{14}$. However, employing an HG 1,0 mode in the reference path in addition to the currently used HG 0,0 mode improves the number of multiplexing by a factor of 2 . Although more area is needed due to the larger dimensions of higher order HG modes, the theoretical recording density still increases by about 1.33. Additionally, the reference path optics do not require substantial modifications. As seen in Fig. 2, the mode diameter along the horizontal direction increases by factor of 1.77 while the vertical dimension is unchanged. An ideal HG 1,0 has a 1/e field radius $\sim 1.5$ times larger than the HG 0,0 beam, so it is clear that the experimental beam is wider than is should be in theory. This may be a result of the phase error mentioned earlier or the fact that our FT geometry does not place the phase plate at the front focal plane or surface of the mode matching lens ${ }^{15}$. Experimentally, storage density increases by factor of $2 / 1.77=1.13$, but in theory increases by $2 / 1.5=1.33$. This seems like a small increase until we consider the trend as higher order modes are added, and the growth in beam size more closely matches the square root approximation ${ }^{13}$. The number of multiplexing increases as the maximum mode number plus one, and mode size increases like the square root of the maximum mode number, so the storage density will increase with the number of modes used as shown in Fig. 8, allowing for a factor of 2.1 storage density increase when five modes are used.

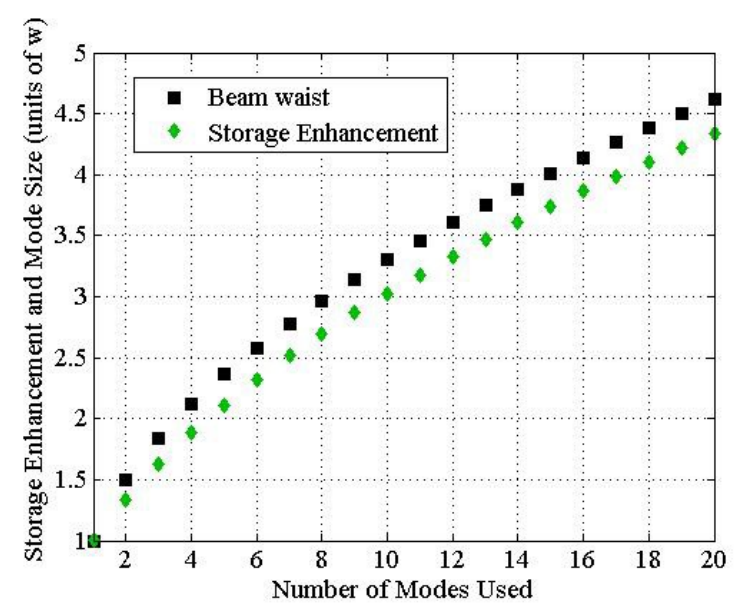

Fig. 8. Maximum mode size (units of Gaussian beam 1/e field radius) as a function of the number of modes used, and storage density enhancement as a function of number of modes used.

While mode multiplexing adds another DOF to HDSS design to increase data density, combining it with angular multiplexing raises the question of angular Bragg selectivity for mode multiplexed holograms. The effects of Gaussian apodization are known to reduce the height of the side lobes in angular multiplexing while increasing the angular width of the Bragg selectivity relative to plane wave reference beams ${ }^{9,16}$. The width of the Bragg selectivity decreases as the beam diameter increases, so we would expect higher order modes to create narrower Bragg selectivities relative to Gaussian recording. Thus, overall, Bragg selectivity for higher order mode multiplexing does not negatively affect storage density, but further investigation is required before mode multiplexing can be implemented in commercial systems. Particularly, the optimum number of reference modes must be investigated while accounting for the tradeoffs in 
beam dimensions and Bragg selectivity, because higher order modes provide diminishing returns on storage density according to Fig. 8.

Bragg selectivity aside, orthogonal mode multiplexing has another advantage when paired with angular multiplexing. Angular multiplexed volume holographic data storage has an issue with beam fanning, which can hinder the use of pseudo-phase conjugate readout when present in the reference arm, but the use of orthogonal reference beams causes the total beam fanning effect to be divided over the all of the reference beams used. Since the reference beams are orthogonal, the beam fanning caused by the self-interference of the reference beams is also matched to the reference beam that created those patterns. Thus beam fanning caused by an HG 1,0 reference is separate from fanning caused by an HG 0,0 reference. This orthogonality of fanning means that fanning of the reference beam should be reduced by a factor equal to or greater than the number of reference beams used.

While reference beam size is a major consideration for mode multiplexing, such considerations are mitigated in orthogonal phase code multiplexing when using codes generated by a Walsh-Hadamard transform ${ }^{10}$. The experimental setup of Fig. 5 is not immediately compatible with arbitrary orthogonal phase coded reference beams, but an appropriate choice of cavity and coupling optics could be made to accommodate a subset of such beams. Thus, the development of a cavity recording scheme which supports a more diverse family of phase codes would remove the concern regarding cavity mode size.

\section{CONCLUSIONS}

Optical cavities in the reference arm make it possible to improve HDSS recording efficiencies, speeds, and data densities when applied with simple Gaussian reference beams. Cavity eigenmodes provide an additional degree of freedom to angular multiplexing for HDSS to increase the recording density. Cavity enhanced, eigenmode and angular multiplexing of image bearing holograms is experimentally demonstrated. Currently, single page cross-talks of $\sim 2.5 \%$ for unenhanced holograms, and $\sim 10 \%$ for cavity mode-angular hybrid multiplexing is confirmed. The total number of multiplexing is increased by the number of eigenmodes supported by the cavity. The trade-off in reference beam area vs. number of multiplexing pages provides diminishing returns on storage density for higher order modes as the beam dimensions increase; however, the use of the first five modes would grant double the storage density. To further increase the recording density, challenges such as mode conversion fidelity, cavity mode matching, and trade-off balancing between angular Bragg spacing and mode size of higher order cavity eigenmodes have to be addressed. Optimization aside, this demonstration provides a pathway to a more complete usage of the available degrees of freedom of three dimensional optical data storage towards the theoretical storage density limit, $1 / \lambda^{3}$ bits $/ \mathrm{inch}^{3}$.

\section{ACKNOWLEDGMENTS}

We would like to thank Hitachi, Ltd. for the use of their Holoeye LC 2012 spatial light modulator.

\section{REFERENCES}

[1] “White Paper: Archival Disc Technology.”, July 2015, $<$ http://panasonic.net/avc/archiver/pdf/E_WhitePaper_ArchivalDisc_Ver100.pdf $>$.

[2] Zhang, J., Čerkauskaite, A., Drevinskas, R., Patel, A., Beresna, M.., Kazansky, P. G., "Eternal 5D data storage by ultrafast laser writing in glass," Proc. SPIE 97360 U (2016).

[3] Anderson, K., Ayres, M., Askham, F.., Sissom, B., "Holographic Data Storage: Science Fiction or Science Fact," Proc. SPIE 9201, 920102 (2014).

[4] van Heerden, P. J., “Theory of Optical Information Storage in Solids,” Appl. Opt. 2(4), 393 (1963).

[5] Ayres, M. R., Anderson, K., Askham, F., Sissom, B.., Urness, A. C., "Holographic data storage at 2+ Tbit/in"," Proc. SPIE 9386, 93860G (2015).

[6] Sinha, A.., Barbastathis, G., "Resonant holography," Opt. Lett. 27(6), 385-387 (2002).

[7] Miller, B. E.., Takashima, Y., "Cavity techniques for holographic data storage recording," Opt. Express 24(6), 6300 (2016). 
[8] Bashaw, M. C., Heanue, J. F., Aharoni, A., Walkup, J. F.., Hesselink, L., "Cross-talk considerations for angular and phase-encoded multiplexing in volume holography,” J. Opt. Soc. Am. B 11(9), 1820 (1994).

[9] Tian, K., "Three dimensional (3D) optical information processing," Thesis, Massachusetts Institute of Technology (2006).

[10] Denz, C., Pauliat, G., Roosen, G.., Tschudi, T., "Volume hologram multiplexing using a deterministic phase encoding method," Opt. Commun. 85(2-3), 171-176 (1991).

[11] Kühn, J.., Patapis, P., "Digital adaptive coronagraphy using SLMs: promising prospects of a novel approach, including high-contrast imaging of multiple stars systems," Proc. SPIE 99122M, R. Navarro and J. H. Burge, Eds. (2016).

[12] “HOLOEYE Photonics AG» LC 2012 Spatial Light Modulator (transmissive).”, <http://holoeye.com/spatiallight-modulators/lc-2012-spatial-light-modulator/> (12 October 2016 ).

[13] Siegman, A. E., "Lasers," [Lasers], University Science Books, Mill Valley, CA, 413-426, 642-648, 690 (1986).

[14] Hoshizawa, T., Shimada, K., Fujita, K.., Tada, Y., "Practical angular-multiplexing holographic data storage system with 2 terabyte capacity and 1 gigabit transfer rate," Jpn. J. Appl. Phys. 55(9S), 09SA06 (2016).

[15] Goodman, J. W., "Introduction to Fourier Optics," [Introduction to Fourier Optics], Roberts \& Company Publishers, Greenwood Village Colorado, 107-108 (2005).

[16] Dai, F.., Gu, C., "Effect of Gaussian references on cross-talk noise reduction in volume holographic memory," Opt. Lett. 22(23), 1802 (1997). 